\title{
Effects of unpaired 1 gene overexpression on the lifespan of Drosophila melanogaster
}

\author{
Alexey Moskalev ${ }^{1,2,3^{*}}$, Ekaterina Proshkina ${ }^{2}$, Alex Zhavoronkov ${ }^{4}$ and Mikhail Shaposhnikov ${ }^{2}$ \\ From 11th International Multiconference "Bioinformatics of Genome Regulation and StructurelSystems Biology" - BGRS\SB- \\ 2018
}

Novosibirsk, Russia. 20-25 August 2018

\begin{abstract}
Background: The JAK/STAT signaling pathway is involved in many aging-related cellular functions. However, effects of overexpression of genes controlling JAK/STAT signal transduction on longevity of model organisms have not been studied. Here we evaluate the effect of overexpression of the unpaired 1 (upd1) gene, which encodes an activating ligand for JAK/STAT pathway, on the lifespan of Drosophila melanogaster.

Results: Overexpression of upd 1 in the intestine caused a pronounced shortening of the median lifespan by 54.1-18.9\%, and the age of $90 \%$ mortality by $40.9-19.1 \%$ in males and females, respectively. In fat body and in nervous system of male flies, an induction of upd 1 overexpression increased the age of $90 \%$ mortality and median lifespan, respectively. An increase in upd 1 expression enhanced mRNA levels of the JAK/STAT target genes domeless and Socs36E.

Conclusions: Conditional overexpression of upd 1 in different tissues of Drosophila imago induces pro-aging or prolongevity effects in tissue-dependent manner. The effects of upd 1 overexpression on lifespan are accompanied by the transcription activation of genes for the components of JAK/STAT pathway.
\end{abstract}

Keywords: Lifespan, Drosophila melanogaster, JAKSSTAT signaling pathway, Unpaired ligand, Mifepristone

\section{Background}

The JAK/STAT (Janus kinase/signal transducers and activators of transcription) signaling pathway is involved in many aging-related functions, including cell proliferation, differentiation, survival, apoptosis, cell senescence, state of heterochromatin, and expression of a variety of genes [1-6]. In addition, the JAK/STAT pathway coordinates processes of stem cell proliferation and differentiation in Drosophila intestine [7-9], Malpighian tubules [10], and testis niche [11], thereby establishing tissue homeostasis. JAK/STAT signaling is an important component of the conserved transcriptional network driving myogenesis [12]. The expression levels of JAK/STAT

\footnotetext{
* Correspondence: amoskalev@ib.komisc.ru

'Engelhardt Institute of Molecular Biology, Russian Academy of Sciences, Moscow 119991, Russia

${ }^{2}$ Institute of Biology, Komi Scientific Center, Ural Division, Russian Academy of Sciences, Syktyvkar 167982, Russia

Full list of author information is available at the end of the article
}

signal pathway proteins are variable throughout the organism lifespan, and tend to decline with age [13].

In Drosophila melanogaster, the unpaired 1 (upd1) gene encodes the most potent ligand for the JAK/STAT signaling pathway [14, 15]. Earlier, Boyle et al. demonstrated that the expression of upd in the stem cells of male gonad niche decreases at the old age proportionally to a decline of stem cell population [16]. Overexpression of upd in gonads of old males impedes the degeneration of germ line stem cells [16]. Thus, literature data suggest a connection between the processes of aging and JAK/ STAT signal pathway activity. At the same time, the effects of overexpression of genes, which encode cytokines controlling the JAK/STAT signaling pathway, on the lifespan have not been studied.

The purpose of the present work is to elucidate whether conditional overexpression of updlin specific tissues (intestine, fat body, and nervous system) affects longevity of Drosophila melanogaster imago.

(c) The Author(s). 2019 Open Access This article is distributed under the terms of the Creative Commons Attribution 4.0 International License (http://creativecommons.org/licenses/by/4.0/), which permits unrestricted use, distribution, and 


\section{Results}

Effects of the upd1 gene overexpression on the lifespan In order to analyze the lifespan effect of the overexpression of cytokines that control JAK/STAT signaling, we conditionally activated the overexpression of the upd1 gene in the intestine, fat body, and nervous system throughout the imago stage.

Overexpression of upd1 in the fly intestine caused a statistically significant decrease in the lifespan of both sexes $(p<0.001)$ (Table 1 , Fig. 1a and b). In males and females, the median lifespan was reduced by 54.1 and $18.9 \%$; the age of $90 \%$ mortality decreased by 40.9 and $19.1 \%$, respectively. Males and females with upd1 overexpression in intestinal cells exhibited higher rates of initial $\left(R_{0}\right)$ and age-dependent $(\alpha)$ mortality (Table 1 ).

In males and females, conditional overexpression of the upd 1 gene in fat body cells (Table 1, Fig. 1c and d) did not produce a statistically significant effect on the median lifespan $(p>0.05)$. At the same time, males demonstrated an increase of $90 \%$ mortality age (by $7.5 \%, p<0.0001$ ). In flies of both sexes, a slight decrease of the age-dependent mortality rate was detected (Table 1).

Conditional activation of upd1 overexpression in nervous system cells (Table 1, Fig. 1e and f) caused altered survival measures. Neuron-specific overexpression of upd1 led to a statistically significant increase of the median lifespan (by $31 \%, p<0.001$ ) in males only. However, the effect of the median lifespan enhancement was accompanied by a decrease in mortality rate doubling time (MRDT) (by 32.1\%) and accelerated aging (Table 1).
Quantification of the upd1, dome, and Socs36E genes expression in different tissues

Analysis of relative expression levels in different imago tissues using the qRT-PCR method confirmed activation of the upd1 gene expression in response to mifepristone treatment (Fig. 2). In male intestinal cells, expression of the upd1 gene was enhanced 25-fold; in females, 6-fold (Fig. 2a). In fat body cells, expression of the upd1 gene increased 2-fold in males and 4-fold in females (Fig. 2b). In nervous system cells, a 1.5-fold enhancement of expression was observed in males, and a 5 -fold increase in females (Fig. 2c).

To clarify whether the lifespan alteration in flies overexpressing upd1 is associated with activation of the JAK/ STAT signaling pathway, we analyzed the expression level of domeless and Suppressor of cytokine signaling at $36 E$ (Socs36E), which are the JAK/STAT target genes $[17,18]$. We found that the activation of upd1 expression led to a 1.5-3-fold increase in the levels of domeless in all studied tissues, and a 1.5-2.5-fold increase in the levels of Socs $36 E$ mRNA in the fat body and nervous system, respectively (Fig. 3). These data suggest that overexpression of upd 1 transcription is accompanied by the induction of JAK/STAT signaling components and may influence the Drosophila lifespan through this pathway.

\section{Histology of intestine}

To find whether the lifespan decreasing in UAS-upd1/ TIGS-2 flies within the digestive system is caused by disruption of intestinal tissue homeostasis, we carried out the histological study. The histological observation of intestines revealed that the mifepristone treatment

Table 1 Effects of conditional (RU486-induced) upd1 gene overexpression in intestinal cells (TIGS-2), fat body cells (Switch1.32) and nervous system cells (ELAV-GS) on the lifespan of imago Drosophila melanogaster

\begin{tabular}{|c|c|c|c|c|c|c|c|c|c|c|c|c|c|}
\hline Genotype & Variant & Sex & $M$ & $d M$ & Cox-Mantel test & $90 \%$ & d90\% & Wang-Allison test & MRDT & dMRDT & $a$ & $R_{0}$ & $n$ \\
\hline UAS-upd/TIGS-2 & $-R \cup 486$ & $\hat{\sigma}$ & 37 & & & 44 & & & 5.5 & & 0.12519 & 0.00094 & 345 \\
\hline UAS-upd/TIGS-2 & $+\mathrm{RU} 486$ & $\widehat{0}$ & 17 & -54.1 & $p<0.001$ & 26 & -40.9 & $p<0.001$ & 5.5 & -0.3 & 0.12555 & 0.00934 & 454 \\
\hline UAS-upd/TIGS-2 & $-R \cup 486$ & 우 & 37 & & & 47 & & & 5.6 & & 0.12277 & 0.00087 & 423 \\
\hline UAS-upd/TIGS-2 & $+\mathrm{RU} 486$ & q & 30 & -18.9 & $p<0.001$ & 38 & -19.1 & $p<0.001$ & 4.9 & -12.4 & 0.14013 & 0.0015 & 470 \\
\hline UAS-upd/Switch1.32 & $-R \cup 486$ & $\hat{0}$ & 62 & & & 67 & & & 6.3 & & 0.10991 & 0.00013 & 283 \\
\hline UAS-upd/Switch1.32 & $+\mathrm{R} \cup 486$ & $\widehat{0}$ & 61 & -1.6 & $p>0.05$ & 72 & 7.5 & $p<0.0001$ & 7.3 & 16.4 & 0.09445 & 0.00023 & 312 \\
\hline UAS-upd/Switch1.32 & $-R \cup 486$ & q & 59 & & & 68 & & & 5.6 & & 0.12379 & 0.00007 & 283 \\
\hline UAS-upd/Switch1.32 & $+R \cup 486$ & 우 & 57 & -3.4 & $p>0.05$ & 68 & 0.0 & $p>0.05$ & 6.5 & 15.9 & 0.10679 & 0.00019 & 336 \\
\hline UAS-upd/ELAV-GS & $-R \cup 486$ & $\hat{0}$ & 43.5 & & & 61 & & & 8.9 & & 0.07795 & 0.0016 & 449 \\
\hline UAS-upd/ELAV-GS & $+\mathrm{R} \cup 486$ & $\hat{0}$ & 57 & 31.0 & $p<0.001$ & 64 & 4.9 & $p>0.05$ & 6.0 & -32.1 & 0.11475 & 0.00016 & 417 \\
\hline UAS-upd/ELAV-GS & $-R \cup 486$ & q & 52 & & & 60 & & & 6.5 & & 0.10705 & 0.00033 & 448 \\
\hline UAS-upd/ELAV-GS & $+R \cup 486$ & 우 & 52 & 0.0 & $p>0.05$ & 61 & 1.7 & $p>0.05$ & 6.4 & -0.7 & 0.10776 & 0.00031 & 462 \\
\hline
\end{tabular}

RU486 - control, without mifepristone; +RU486 - overexpression, with mifepristone; $\delta^{-}$- males; 9 - females; M - (days); $90 \%$ - age of $90 \%$ mortality (days); MRDT - mortality rate doubling time (days); $a$ and $R_{0}$ - Gompertz equation parameters; $n$ - sample size. $d M$, d90\%, dMRDT - the differences in median lifespan, age of $90 \%$ mortality and MRDT estimated between -RU486 and + RU486 variants (\%) 

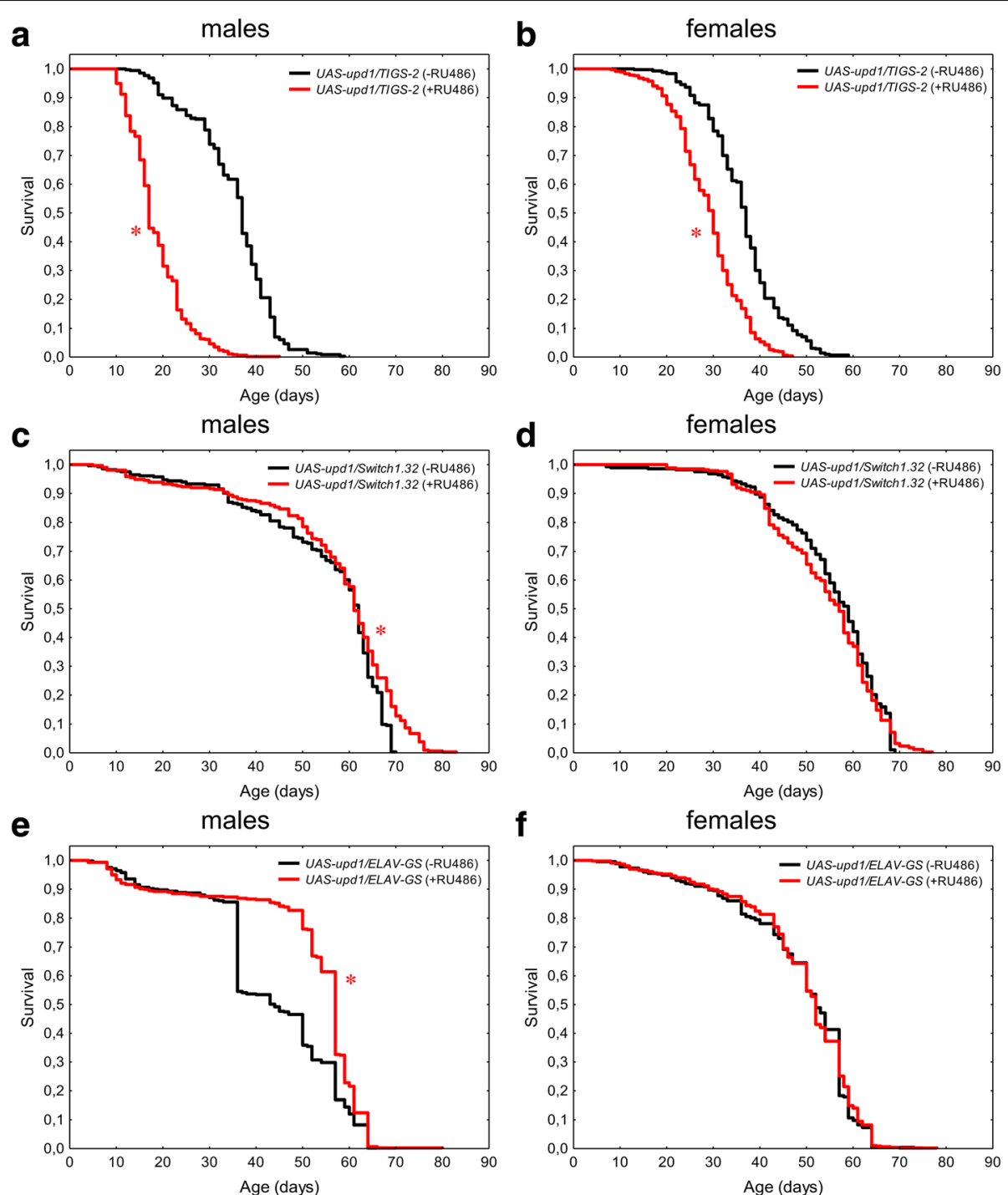

Fig. 1 Effects of the upd 1 gene overexpression in the intestines $(\mathbf{a}, \mathbf{b})$, fat body $(\mathbf{c}, \mathbf{d})$ and nervous system $(\mathbf{e}, \mathbf{f})$ on the lifespan of Drosophila melanogaster males $(\mathbf{a}, \mathbf{c}, \mathbf{e})$ and females $(\mathbf{b}, \mathbf{d}, \mathbf{f})$. The results of two experimental replications are merged. ${ }^{*} p<0.001$, Kolmogorov-Smirnov test. The presented data correspond to the Table 1

induced progressive dysplasia of gut epithelium in UAS-upd1/TIGS-2 females at ages from 1 to 10 days (Fig. 4). This disruption of intestinal tissue homeostasis may result in the increasing mortality rates of flies with upd1 overexpression in intestine.

\section{Discussion}

The JAK/STAT pathway is one of the few signaling cascades that are evolutionarily conserved in multicellular animals from flies to humans both on the structural and functional levels $[1,19]$. JAK/STAT signaling is involved in the transduction of intercellular biochemical signals essential for the development and the maintenance of homeostasis [19]. In mammals, the JAK/STAT pathway is activated by a broad spectrum of cytokines and growth factors, which stimulate cell proliferation, differentiation, migration, and apoptosis [19]. These cellular processes are critical for hematopoiesis, immune development, adipogenesis, sexually dimorphic growth, and aging. The expression levels of these signal pathway proteins do fluctuate throughout the lifespan of an organism, but decrease with age in macrophages, natural killer cells, hippocampus, and myocardium [13].

In contrast to mammals, Drosophila melanogaster genome encodes for only three ligands with JAK/STAT activating properties. These ligands are encoded by the unpaired gene family that includes upd1, upd2, and upd3 [15]. The reduced expression of upd1 in old Drosophila males correlates with an overall decrease of germ line stem cells number [16]. Thus, data obtained 


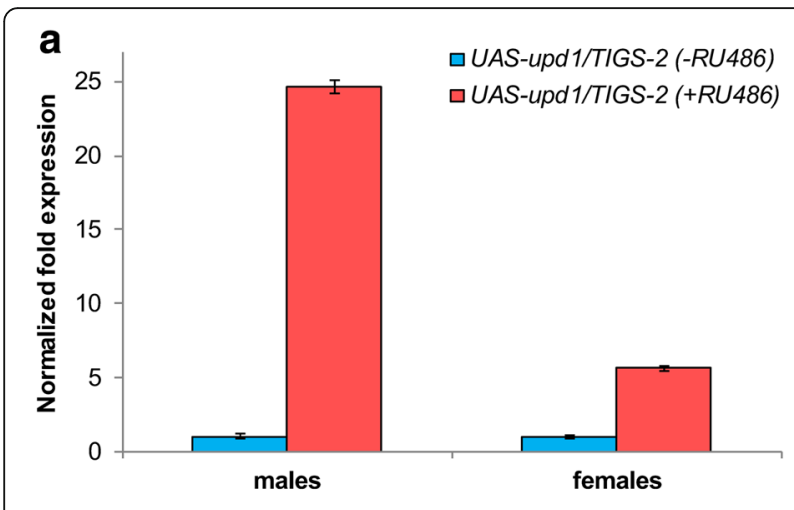

b

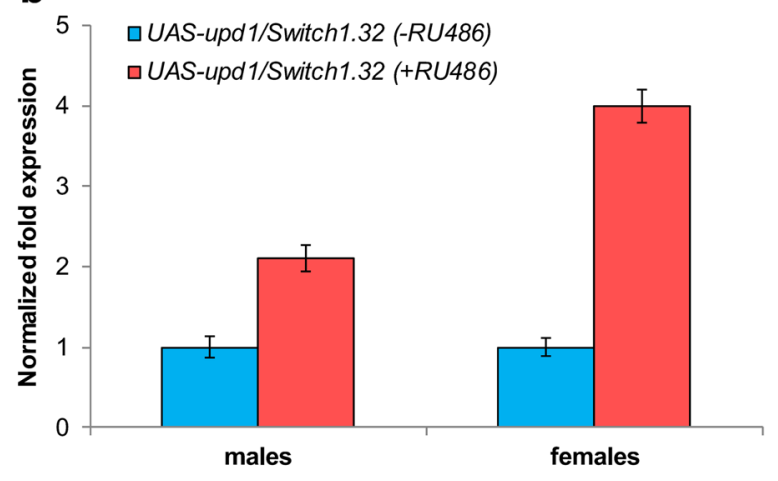

c

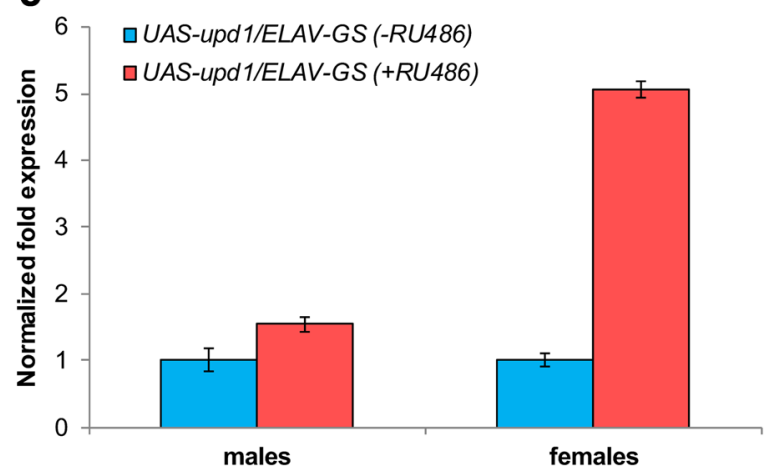

Fig. 2 Relative expression levels of the updl gene in Drosophila melanogaster imago tissues activated by tissue-specific drivers: a intestines (TIGS-2); b fat body (Switch 1.32); c nervous system (ELAV-GS)

for mammals and flies point towards alterations of the JAK/STAT signaling activity in aging cells and organs. However, the effect of the JAK/STAT signal pathway overactivation on the organism lifespan was not studied previously. At the same time, a number of recent publications demonstrates lifespan extension in flies with overexpression of genes, which enhance resistance to oxidative stress (SOD1) [20] and survival under stress conditions $(F O X O)[21,22]$, control repair of proteins (PCMT and dmsrA) [23, 24] and DNA repair [25], increase female fecundity at late ages (hebe and magu) [26], and reduce insulin/IGF-1 (UCP) [27] or TOR kinase (dTsc1, $d T s c 2$ or dominant-negative forms of $d T O R$ or $d S 6 K)$ [28] signaling pathways. Loss of function and knockdown of genes regulating the insulin/IGF-1 signaling pathway during growth (Lnk) [29], energy metabolism (Indy and mitochondrial electron transport chain genes) [30,31], or affecting intracellular signaling pathways (mth) [32] extend Drosophila lifespan, as well.

The crucial tissues for the most of longevity genes activity are intestinal [30], adipose [22, 28], and nervous $[20,24,27,31]$ tissues, which are the principal sites of metabolism in the fly.

Thus, in order to investigate the effect of the JAK/ STAT signal pathway overactivation on the organism lifespan, we activated overexpression of the upd1 gene that is the most potent ligand for the JAK/STAT signaling pathway [15] in Drosophila melanogaster in some indispensable-to-longevity imago tissues (intestine, fat body, nervous system). The use of conditional mifepristone-activated tissue-specific Gene-Switch drivers enables us to control not only the tissue, but also the stage of transgene expression induction (throughout the imago lifespan), as well as to exclude the effects of different genetic backgrounds on the lifespan [33]. However mifepristone has been reported to affect lifespan of males and mated females differently. It increases the longevity of mated females but not males by various mechanisms including modulation of feeding rate and innate immune response [34-36]. Sex-specific differences in the response to mifepristone treatment may explain the different effects of the upd 1 overexpression in the fat body and the nervous system in males and females. The positive effects of mifepristone feeding and upd1 overexpression in these tissues are indistinguishable in mated females. At the same time, the strongly pronounced negative effect of the upd1 overexpression in the intestine on lifespan does not overlap with the positive effect of mifepristone.

In addition the Gene-Switch drivers which were used in our study has been reported to demonstrate sex-specific differences in the expression levels (both temporal and spatial) in the presence of the inducer and leaky expression in non-target tissues in the absence of the inducer [37], which makes any meaningful comparisons between the males and females very complicated.

As shown by a series of studies, intestines of old flies and flies exposed to stress exhibit depletion of epithelium accompanied by excessive proliferation and aberrant differentiation of stem cells [38, 39]. Moderate activation of JNK signaling in intestinal cells in response to stress results in differentiation anomalies and in substantial decrease of a lifespan [38]. Maintaining of tissue homeostasis involves the JAK/STAT signaling pathway, too [7]. 


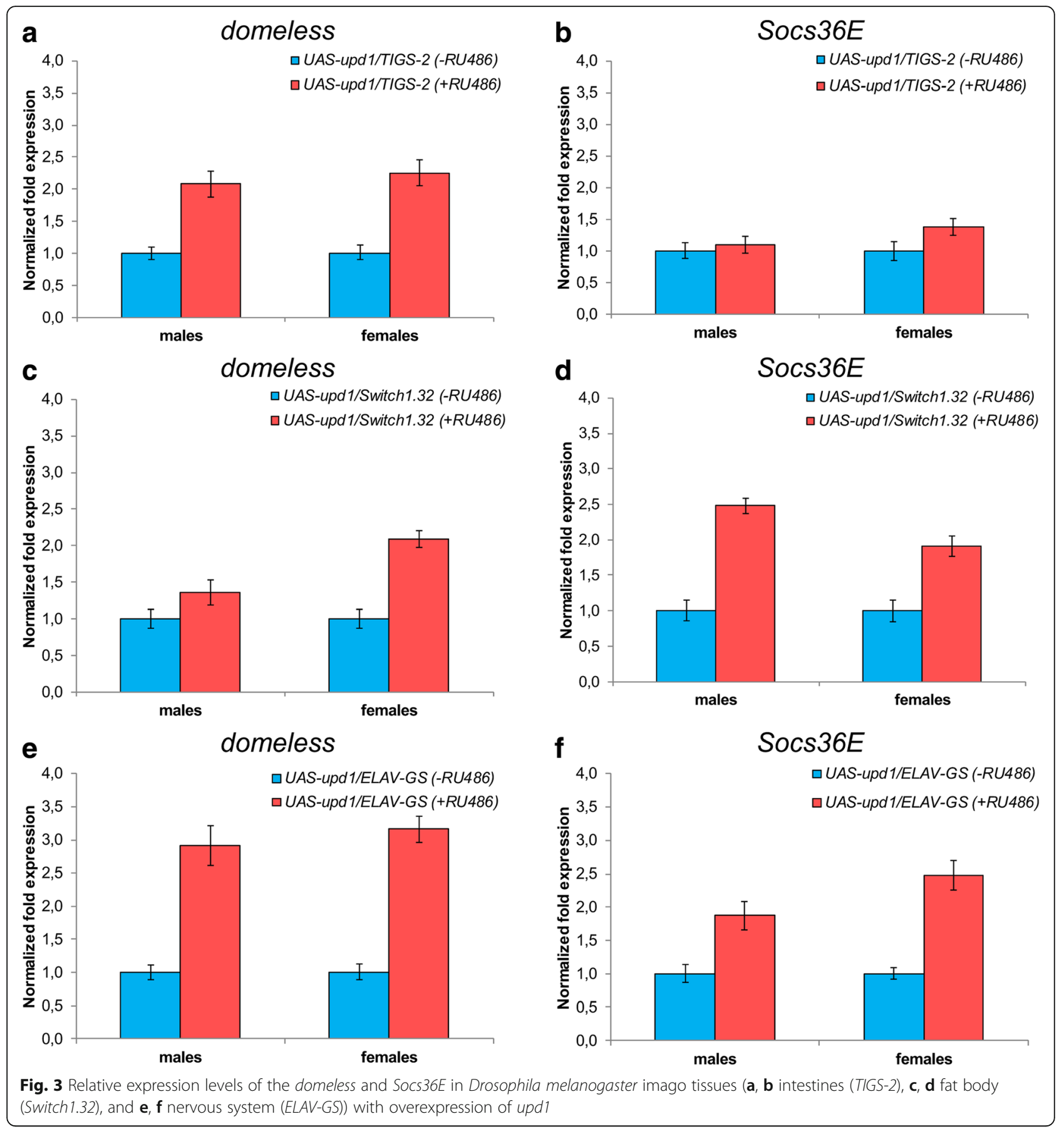

We have shown that induction of upd1 overexpression in the intestine causes significant lifespan shortening in individuals of both sexes. Accelerated aging of the organism is seen upon both strong induction of upd 1 expression in males (25-fold), moderate induction in females (6-fold), and weak activation of the domeless gene (2-fold). This effect could be caused by disruption of intestinal tissue homeostasis resulting from excessive activation of JAK/STAT. As it was shown by $\mathrm{Li}$ et al., chronic activation of JAK/
STAT signaling in the gut of aging Drosophila is associated with age-related metaplasia, commensal dysbiosis and functional decline of the gastrointestinal tract. Accordingly, inhibition of JAK/STAT signaling in the stomach-like copper cell region prevents these negative changes and extends lifespan of flies [40]. Histological observations of female intestines display that upd1 overexpression results in dysplasia of gut epithelium, which may be the cause of lifespan decrease to a considerable extent. 


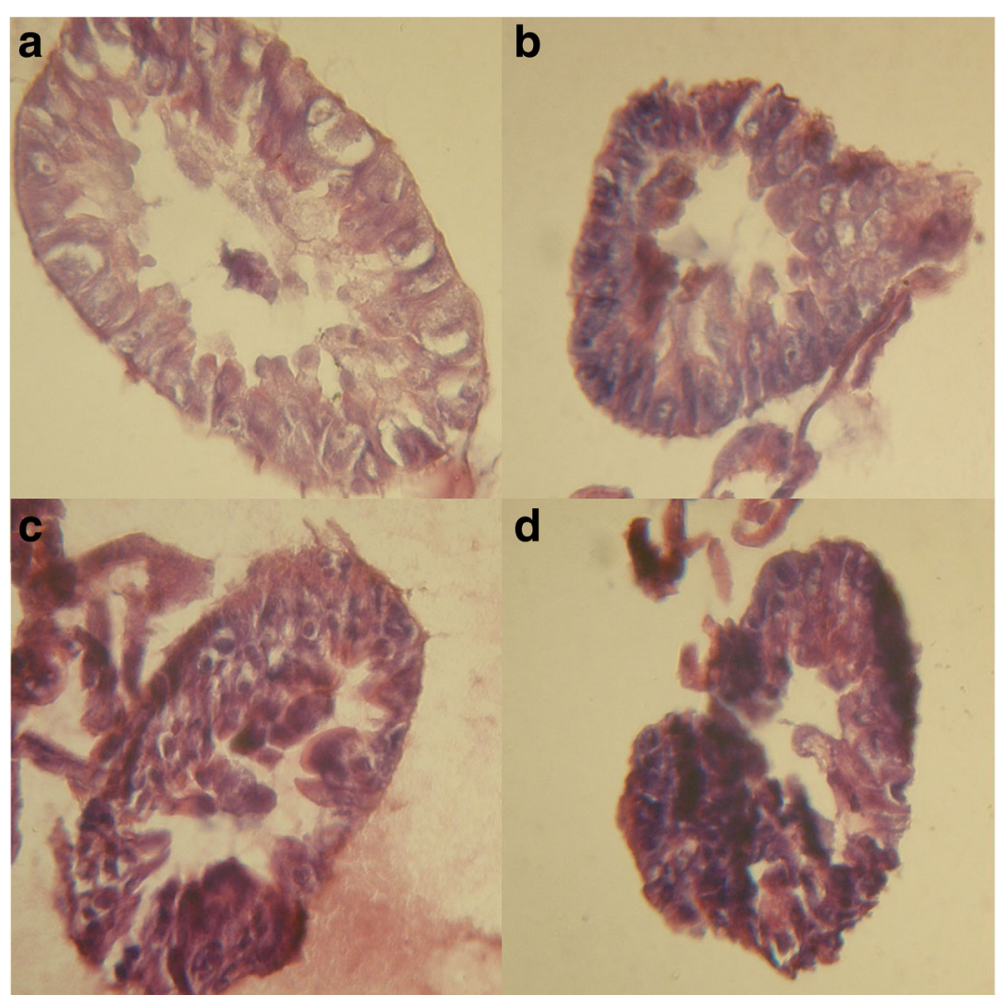

Fig. 4 Mifepristone induces dysplasia of gut epithelium in in UAS-upd1/TIGS-2 females. Transverse sections through the intestine of: a 1 day old (-RU486), b 10 days old (-RU486), c 1 day old (+RU486), d 10 days old (+RU486). All magnitudes $\times 800$

Agaisse et al. showed that activation of JAK/STAT pathway in the Drosophila fat body by upd3 ligand facilitates the formation of immune response to septic shock [41]. It is also known that JAK/STAT signaling demonstrates anti-apoptotic activity [42]. An increase of apoptosis levels in postmitotic tissues with age can lead to a decline of functional activity of the tissue as a result of irretrievable cell loss. Previously, Zheng et al. have shown that under normal conditions, fly aging is accompanied by a steady increase of apoptosis level in muscle and fat cells [43]. Therefore, an increase of the age of $90 \%$ mortality in male individuals at weak level (2-fold) of $u p d 1$ overexpression in the fat body may be caused by activation of the immune system and anti-apoptotic activity.

It is of note that sustained production of macrophagederived upd3 in response to chronic lipid-rich diet was associated with activation of JAK-STAT signaling, increased fat storage, reduced insulin sensitivity, hyperglycemia, and a shorter lifespan of Drosophila flies [44]. Upd2 is produced by the Drosophila fat body in response to dietary fats and sugars and promote secretion of Drosophila insulin-like peptides [45]. The opposite effects of different unpaired ligands on the lifespan and aging may be associated with their different functions.
We also demonstrated that the low level of upd1 overexpression (1.5-fold) in the nervous system of males was accompanied by the induction of JAK/STAT signaling components domeless (3-fold) and Socs36E (2-fold) and led to increase of median lifespan. As it was shown by Chiba et al., age-dependent deterioration in the JAK2/ STAT3 axis plays a critical role in the pathogenesis of Alzheimer's disease [46]. The activity of the JAK/STAT pathway has been shown to be involved in synaptic plasticity in the mammalian brain [47]. Therefore, the overexpression of upd1 and activation of the JAK/STAT pathway can potentially prevent neurodegeneration and age-related deficits in synaptic plasticity contributing to longevity.

\section{Conclusions}

Thus, conditional overexpression of the upd1 gene encoding a JAK/STAT signaling ligand results in alteration of the Drosophila lifespan depending on the tissue specificity of overexpression. The effects of upd 1 overexpression on lifespan are accompanied by the transcription activation of the JAK/STAT target genes domeless and Socs36E. As the JAK/STAT pathway is evolutionarily conserved it may be possible to discover compounds that fit the criteria of geroprotector [48]. In our prior 
work we explored the effects of a variety of interventions on the Drosophila lifespan [49-53]. The new pathway analysis algorithms enable us to identify the pharmacological interventions for modulation of the levels of expression of individual genes and networks. In our future work we plan to test the compounds from DrugAge [54] and Geroprotectors.org [55] and other libraries potentially modulating upd, domeless and Socs36E on the lifespan of Drosophila and other organisms.

\section{Material and methods}

\section{Drosophila melanogaster lines}

UAS-upd1 (genotype: $w^{1118}$; UAS-upd1/CyO): transformed line in which expression of an additional copy of the unpaired 1 gene is controlled by UAS promoter (kindly provided by Dr. James Castelli-Gair Hombría, Universidad Pablo de Olavide, Sevilla, Spain) [56].

TIGS-2: Gene-Switch driver line containing mifepristone-inducible GAL4 in intestine cells (kindly provided by Dr. Laurant Seroude, Queen's University, Kingston, Canada) [37].

Switch1.32 (genotype: $w^{1118} ; \mathrm{P}\left\{w^{+m W . h s}=\right.$ Switch1 $\}$ bun ${ }^{\text {S- }}$ witch $1.32 / C y O)$ : driver line containing mifepristone-inducible GAL4 in imago head fat body cells (kindly provided by Dr. Marc Tatar, Brown University, Providence, Rhode Island, USA) [33].

ELAV-GS (genotype: $y$ w; P\{ELAV-GeneSwitch $\}$ ): GeneSwitch driver line containing mifepristone-inducible GAL4 in nervous system cells (kindly provided by Dr. Haig Keshishian, Yale University, New Haven, Connecticut, USA) [33].

\section{Activation of the upd 1 gene overexpression}

Overexpression of the upd1 gene in Drosophila melanogaster was activated using the GAL4/UAS system. Crossing of UAS-upd1 line females with Gene-Switch system driver line males (Switch1.32, ELAV-GS, and TIGS-2) resulted in UAS-upd1/TIGS-2, UAS-upd1/Switch1.32, and $U A S$-upd1/ELAV-GS genotypes in $\mathrm{F}_{1}$. After eclosion males and females were allowed to mate during the $24 \mathrm{~h}$ and then separated according to sex. Overexpression was conditionally activated by feeding the $F_{1}$ imagoes with yeast paste supplemented with $25 \mu \mathrm{g} / \mathrm{ml}$ mifepristone (RU486, Sigma-Aldrich, USA). Flies were treated with RU486 throughout life of adults. Control animals were fed yeast paste without RU486.

\section{Lifespan analysis}

Control and experimental flies were maintained at $25 \pm$ $0.5^{\circ} \mathrm{C}$ under a $12 \mathrm{~h} \mathrm{light} / 12 \mathrm{~h}$ dark cycle and at densities of 30 same sex and age flies per vial with sugar-yeast medium [57] covered with a yeast paste. Flies were transferred to fresh medium three times per week. Lifespan was analyzed daily, separately for males and females. Every variant of the experiment was analyzed in two replications. Survival functions were estimated using the Kaplan-Meier procedure and plotted as survival curves. Median lifespan and the age of $90 \%$ mortality (maximum lifespan) were calculated. The initial $\left(\mathrm{R}_{0}\right)$ and age-dependent $(\alpha)$ mortality (parameters of the Gompertz equation $\left.\left(\mu(x)=R_{0} \mathrm{e}^{\alpha x}\right)\right)$ and the mortality rate doubling time $(\mathrm{MRDT}=\ln 2 / \alpha)$ were estimated. Nonparametric methods were used for statistical processing of results. Comparison of survival functions was done using the modified Kolmogorov-Smirnov test [58]. The Mantel-Cox test [59] was used to evaluate the statistical significance of medial lifespan differences. To test the statistical significance of differences in age of $90 \%$ mortality (maximum lifespan), the Wang-Allison test was used [60]. Statistical analysis was carried out using Statistica version 6.1, StatSoft, Inc., R, version 2.15.1 (The R Foundation) and OASIS 2: Online Application for Survival Analysis 2 [61].

\section{Quantitative real-time PCR}

To quantify the gene expression levels in different imago tissues, 50 flies were used for mRNA isolation and cDNA synthesis per each experimental variant. The flies were treated by mifepristone during 3-5 days after imago hatching. Expression levels in males and females were estimated separately. Fly heads were excised to estimate the levels of Switch1.32 and ELAV-GS driven expression in head fat body or nervous system, respectively. Intestines were dissected for TIGS-2 driven expression estimation in digestive system. The homogenization of isolated organs using the SilentCrusher S homogenizer (Heidolph Instruments, Germany) was followed by RNA extraction with TRIzol Reagent (Invitrogen, USA), according to the manufacturer's protocol. To assure the absence of RNA samples contamination by DNA, one of the PCRs was performed using $\beta$-Tubulin56D $(\beta T u b 56 D)$ gene primers without reverse transcription. For cDNA synthesis, the Oligo(dT) primer (Invitrogen, USA) and SuperScript III First-Stand Synthesis System reverse transcriptase (Invitrogen, USA) were used according to the manufacturer's protocol. Real-time PCR was carried out using $30 \mu \mathrm{l}$ of reaction mixture containing SYBR Green PCR Master Mix (Applied Biosystems, USA) in 200- $\mu$ l PCR tubes with primers listed in Table 2. According to pcrEfficiency estimation used primers have similar efficiencies for PCR amplification [62]. Real-time PCR reactions were performed on the nucleic acid analyzer ANK-32 (Institute of Analytical Instrumentation of the Russian Academy of Sciences, Russia). All real-time PCR assays were run using the following program: 1) denaturing at $95^{\circ} \mathrm{C}$ for $\left.10 \mathrm{~min} ., 2\right)$ denaturing at $95^{\circ} \mathrm{C}$ for $15 \mathrm{~s}, 3$ ) annealing at $60^{\circ} \mathrm{C}$ for $\left.30 \mathrm{~s}, 4\right)$ elongation 
Table 2 Nucleotide sequences of primers used for quantitative real-time PCR

\begin{tabular}{lllll}
\hline Gene & Forward primer $\left(5^{\prime}-3^{\prime}\right)$ & Reverse primer (5'-3') & Product length (in bp) & Expected efficiency \\
\hline upd1 & agacagccgtcaaccagac & gcttcaaacgcttgttcatc & 141 & $>2$ \\
domeless & tcgctacatgacaacgtgaccgat & acgcacgctaatctcgtacttggt & 132 & $>2$ \\
Socs36E & gggcaaacagaacccagaaaccaa & tccgagctgcattccaataggtga & 189 & $>2$ \\
BTub56D & gcaactccactgccatcc & cctgctcctcctcgaact & 216 & $>2$ \\
\hline
\end{tabular}

at $60^{\circ} \mathrm{C}$ for $\left.30 \mathrm{~s}, 5\right)$ stages $2-4$ were repeated 50 times. Amplification was carried out in separate tubes for each gene. 4-8 measurements were performed per each experimental variant. $\beta$-Tubulin $56 D$ was used as the endogenous reference gene. The relative quantification of the upd1, domeless, and Socs36E expression was carried out according to the $2^{-\Delta \Delta C(T)}$ method [63] and expressed as relative fold change normalized to $\beta$-Tubulin56D. Cycle threshold $\left(C_{\mathrm{T}}\right)$ values were determined using the $A N K 32$ software package, version 1.1 (Institute of Analytical Instrumentation of the Russian Academy of Sciences, Russia). The $C_{T}$ values used for the following analysis were not over than 28 cycles. $\Delta \Delta \mathrm{C}_{\mathrm{T}}$ was calculated as $\Delta \Delta \mathrm{C}_{\mathrm{T}}=\Delta \mathrm{C}_{\mathrm{T}}$ (overexpression of upd1) $-\Delta \mathrm{C}_{\mathrm{T}}$ (no overexpression of upd1), and each value $\Delta \mathrm{C}_{\mathrm{T}}=\mathrm{C}_{\mathrm{T}}$ (target gene) $-\mathrm{C}_{\mathrm{T}}$ (reference gene)

\section{Histology of intestine}

Intestines were dissected and fixed in mixture of $85 \%$ ethanol / 4\% formaldehyde / 5\% acetic acid / $1 \%$ glutaraldehyde for $48 \mathrm{~h}$. Then, fixed organs were paraffin embedded, sectioned, hematoxylin and eosin (H\&E) stained, and analyzed by light microscopy [64]. Images of the intestine of young and 10-day old female flies were acquired and analyzed.

\footnotetext{
Abbreviations

JAK/STAT: Janus kinase/signal transducers and activators of transcription; MRDT: Mortality rate doubling time; Socs36E: Suppressor of cytokine signaling at $36 E$
}

\section{Acknowledgments}

We are grateful to Dr. James Castelli-Gair Hombría (Universidad Pablo de Olavide, Sevilla, Spain), Dr. Laurant Seroude, (Queen's University, Kingston, Canada), Dr. Marc Tatar (Brown University, Providence, Rhode Island, USA), Dr. Haig Keshishian (Yale University, New Haven, Connecticut, USA), and the Drosophila Stock Center (Indiana University, Bloomington, Indiana, USA) for providing the Drosophila melanogaster lines, to Dr. Robert Salomon (Tufts Medical Center, Boston, Massachusetts, USA) for his expert opinion in Drosophila intestine histology. We thank the Insilico Medicine and MIPT for assistance in data analysis.

\section{Funding}

The study was carried out within the framework of the state task on themes "Molecular-genetic mechanisms of aging, lifespan, and stress resistance of Drosophila melanogaster", state registration № AAAA-A18-118011120004-5 and "A combination of factors of different nature (low temperature, lack of lighting, restrictive diet, and geroprotector) to maximize the lifespan of Drosophila. Complex UrB RAS Programme" № 18-7-4-23, state registration № AAAA-A18-118011120008-3. Publication costs are funded by Insilico Medicine.

Availability of data and materials Not applicable.

\section{About this supplement}

This article has been published as part of BMC Systems Biology Volume 13 Supplement 1, 2019: Selected articles from BGRSISB-2018: systems biology. The full contents of the supplement are available online at https://bmcsystbiol.biomedcentral.com/articles/supplements/volume-13supplement-1.

\section{Authors' contributions}

AM, MS, EP, AZ wrote the manuscript text. MS, EP carried out the experiments. AM and AZ supervised the text of the manuscript. All authors read and approved the final manuscript.

\section{Ethics approval and consent to participate}

Not applicable.

\section{Consent for publication}

Not applicable.

\section{Competing interests}

Author Alex Zhavoronkov is employed by Insilico Medicine, Inc., a longevity biotechnology and artificial intelligence company. All other authors declare no competing interests.

\section{Publisher's Note}

Springer Nature remains neutral with regard to jurisdictional claims in published maps and institutional affiliations.

\section{Author details}

${ }^{1}$ Engelhardt Institute of Molecular Biology, Russian Academy of Sciences, Moscow 119991, Russia. ${ }^{2}$ Institute of Biology, Komi Scientific Center, Ural Division, Russian Academy of Sciences, Syktyvkar 167982, Russia. ${ }^{3}$ Moscow Institute of Physics and Technology, Dolgoprudny 141700, Russia. ${ }^{4}$ Insilico Medicine, Rockville, MD, USA.

Published: 5 March 2019

\section{References}

1. Arbouzova NI, Zeidler MP. JAK/STAT signalling in Drosophila: insights into conserved regulatory and cellular functions. Development. 2006;133:2605-16.

2. Silver-Morse L, Li WX. JAK-STAT in heterochromatin and genome stability. Jak-Stat. 2013;2:e26090.

3. Xu M, Tchkonia T, Kirkland JL. Perspective: targeting the JAK/STAT pathway to fight age-related dysfunction. Pharmacol Res. 2016;111:152-4.

4. Moskalev A, Zhikrivetskaya S, Shaposhnikov M, Dobrovolskaya E, Gurinovich R, Kuryan O, Pashuk A, Jellen LC, Aliper A, Peregudov A, Zhavoronkov A. Aging chart: a community resource for rapid exploratory pathway analysis of age-related processes. Nucleic Acids Res. 2016;44:D894-9.

5. Moskalev AA, Aliper AM, Smit-McBride Z, Buzdin A, Zhavoronkov A. Genetics and epigenetics of aging and longevity. Cell Cycle. 2014;13:1063-77.

6. Moskalev AA, Shaposhnikov MV, Plyusnina EN, Zhavoronkov A, Budovsky A, Yanai $\mathrm{H}$, Fraifeld VE. The role of DNA damage and repair in aging through the prism of Koch-like criteria. Ageing Res Rev. 2013;12:661-84.

7. Jiang H, Patel PH, Kohlmaier A, Grenley MO, McEwen DG, Edgar BA. Cytokine/Jak/Stat signaling mediates regeneration and homeostasis in the Drosophila midgut. Cell. 2009;137:1343-55.

8. Beebe K, Lee WC, Micchelli CA. JAK/STAT signaling coordinates stem cell proliferation and multilineage differentiation in the Drosophila intestinal stem cell lineage. Dev Biol. 2010;338:28-37. 
9. Liu W, Singh SR, Hou SX. JAK-STAT is restrained by notch to control cell proliferation of the Drosophila intestinal stem cells. J Cell Biochem. 2010;109: 992-9.

10. Singh SR, Liu W, Hou SX. The adult Drosophila malpighian tubules are maintained by multipotent stem cells. Cell Stem Cell. 2007;1:191-203.

11. Singh SR, Zheng Z, Wang H, Oh SW, Chen X, Hou SX. Competitiveness for the niche and mutual dependence of the germline and somatic stem cells in the Drosophila testis are regulated by the JAK/STAT signaling. J Cell Physiol. 2010;223:500-10

12. Liu YH, Jakobsen JS, Valentin G, Amarantos I, Gilmour DT, Furlong EE. A systematic analysis of tinman function reveals Eya and JAK-STAT signaling as essential regulators of muscle development. Dev Cell. 2009;16:280-91.

13. Boengler K, Hilfiker-Kleiner D, Drexler H, Heusch G, Schulz R. The myocardial JAK/STAT pathway: from protection to failure. Pharmacol Ther. 2008;120: 172-85.

14. Harrison DA, McCoon PE, Binari R, Gilman M, Perrimon N. Drosophila unpaired encodes a secreted protein that activates the JAK signaling pathway. Genes Dev. 1998;12:3252-63.

15. Wright VM, Vogt KL, Smythe E, Zeidler MP. Differential activities of the Drosophila JAK/STAT pathway ligands Upd, Upd2 and Upd3. Cell Signal. 2011:23:920-7.

16. Boyle M, Wong C, Rocha M, Jones DL. Decline in self-renewal factors contributes to aging of the stem cell niche in the Drosophila testis. Cell Stem Cell. 2007;1:470-8.

17. Bach EA, Vincent S, Zeidler MP, Perrimon N. A sensitized genetic screen to identify novel regulators and components of the Drosophila janus kinase/ signal transducer and activator of transcription pathway. Genetics. 2003;165: 1149-66.

18. Karsten P, Hader S, Zeidler MP. Cloning and expression of Drosophila SOCS36E and its potential regulation by the JAKJSTAT pathway. Mech Dev. 2002;117:343-6.

19. Rawlings JS, Rosler KM, Harrison DA. The JAK/STAT signaling pathway. J Cell Sci. 2004;117:1281-3

20. Parkes TL, Elia AJ, Dickinson D, Hilliker AJ, Phillips JP, Boulianne GL. Extension of Drosophila lifespan by overexpression of human SOD1 in motorneurons. Nat Genet. 1998;19:171-4.

21. Min KJ, Yamamoto R, Buch S, Pankratz M, Tatar M. Drosophila lifespan control by dietary restriction independent of insulin-like signaling. Aging Cell. 2008;7:199-206.

22. Giannakou ME, Goss M, Junger MA, Hafen E, Leevers SJ, Partridge L. Longlived Drosophila with overexpressed dFOXO in adult fat body. Science. 2004 305:361.

23. Chavous DA, Jackson FR, O'Connor CM. Extension of the Drosophila lifespan by overexpression of a protein repair methyltransferase. Proc Natl Acad Sci U S A. 2001;98:14814-8.

24. Chung H, Kim AK, Jung SA, Kim SW, Yu K, Lee JH. The Drosophila homolog of methionine sulfoxide reductase a extends lifespan and increases nuclear Iocalization of FOXO. FEBS Lett. 2010;584:3609-14.

25. Plyusnina EN, Shaposhnikov MV, Moskalev AA. Increase of Drosophila melanogaster lifespan due to D-GADD45 overexpression in the nervous system. Biogerontology. 2011;12:211-26.

26. Li Y, Tower J. Adult-specific over-expression of the Drosophila genes magu and hebe increases life span and modulates late-age female fecundity. Mo Gen Genomics. 2009;281:147-62.

27. Fridell YW, Hoh M, Kreneisz O, Hosier S, Chang C, Scantling D, Mulkey DK, Helfand SL. Increased uncoupling protein (UCP) activity in Drosophila insulin-producing neurons attenuates insulin signaling and extends lifespan. Aging (Albany NY). 2009;1:699-713.

28. Kapahi P, Zid BM, Harper T, Koslover D, Sapin V, Benzer S. Regulation of lifespan in Drosophila by modulation of genes in the TOR signaling pathway. Curr Biol. 2004;14:885-90.

29. Slack C, Werz C, Wieser D, Alic N, Foley A, Stocker H, Withers DJ, Thornton JM Hafen E, Partridge L. Regulation of lifespan, metabolism, and stress responses by the Drosophila SH2B protein. Lnk PLoS Genet. 2010;6:e1000881.

30. Rogina B, Reenan RA, Nilsen SP, Helfand SL. Extended life-span conferred by cotransporter gene mutations in Drosophila. Science. 2000;290:2137-40.

31. Copeland JM, Cho J, Lo T Jr, Hur JH, Bahadorani S, Arabyan T, Rabie J, Soh J, Walker DW. Extension of Drosophila life span by RNAi of the mitochondrial respiratory chain. Curr Biol. 2009;19:1591-8.

32. Lin YJ, Seroude L, Benzer S. Extended life-span and stress resistance in the Drosophila mutant methuselah. Science. 1998;282:943-6.
33. Roman G, Endo K, Zong L, Davis RL. P\{switch\}, a system for spatial and temporal control of gene expression in Drosophila melanogaster. Proc Natl Acad Sci U S A. 2001;98:12602-7.

34. Landis GN, Salomon MP, Keroles D, Brookes N, Sekimura T, Tower J. The progesterone antagonist mifepristone/RU486 blocks the negative effect on life span caused by mating in female Drosophila. Aging (Albany NY). 2015;7: 53-69.

35. Tower J, Landis GN, Shen J, Choi R, Fan Y, Lee D, Song J. Mifepristone/ RU486 acts in Drosophila melanogaster females to counteract the life spanshortening and pro-inflammatory effects of male sex peptide. Biogerontology. 2017;18:413-27.

36. Yamada R, Deshpande SA, Keebaugh ES, Ehrlich MR, Soto Obando A, Ja WW. Mifepristone reduces food palatability and affects Drosophila feeding and lifespan. J Gerontol A Biol Sci Med Sci. 2017;72:173-80.

37. Poirier L, Shane A, Zheng J, Seroude L. Characterization of the Drosophila gene-switch system in aging studies: a cautionary tale. Aging Cell. 2008;7: 758-70.

38. Biteau B, Hochmuth CE, Jasper H. JNK activity in somatic stem cells causes loss of tissue homeostasis in the aging Drosophila gut. Cell Stem Cell. 2008; 3:442-55

39. Choi NH, Kim JG, Yang DJ, Kim YS, Yoo MA. Age-related changes in Drosophila midgut are associated with PVF2, a PDGFNEGF-like growth factor. Aging Cell. 2008;7:318-34.

40. Li H, Qi Y, Jasper H. Preventing age-related decline of gut compartmentalization limits microbiota Dysbiosis and extends lifespan. Cell Host Microbe. 2016;19:240-53.

41. Agaisse $H$, Petersen UM, Boutros M, Mathey-Prevot B, Perrimon N. Signaling role of hemocytes in Drosophila JAK/STAT-dependent response to septic injury. Dev Cell. 2003;5:441-50.

42. Borensztejn A, Mascaro A, Wharton KA. JAK/STAT signaling prevents excessive apoptosis to ensure maintenance of the interfollicular stalk critical for Drosophila oogenesis. Dev Biol. 2018:438:1-9.

43. Zheng J, Edelman SW, Tharmarajah G, Walker DW, Pletcher SD, Seroude L. Differential patterns of apoptosis in response to aging in Drosophila. Proc Natl Acad Sci U S A. 2005;102:12083-8.

44. Woodcock KJ, Kierdorf K, Pouchelon CA, Vivancos V, Dionne MS, Geissmann F. Macrophage-derived upd3 cytokine causes impaired glucose homeostasis and reduced lifespan in Drosophila fed a lipid-rich diet. Immunity. 2015;42: 133-44.

45. Rajan A, Perrimon N. Drosophila cytokine unpaired 2 regulates physiological homeostasis by remotely controlling insulin secretion. Cell. 2012;151:123-37.

46. Chiba T, Yamada M, Aiso S. Targeting the JAK2/STAT3 axis in Alzheimer's disease. Expert Opin Ther Targets. 2009;13:1155-67.

47. Nicolas CS, Peineau S, Amici M, Csaba Z, Fafouri A, Javalet C, Collett VJ, Hildebrandt L, Seaton G, Choi SL, Sim SE, Bradley C, Lee K, Zhuo M, Kaang BK, Gressens P, Dournaud P, Fitzjohn SM, Bortolotto ZA, Cho K, Collingridge GL. The Jak/STAT pathway is involved in synaptic plasticity. Neuron. 2012;73: 374-90.

48. Moskalev A, Chernyagina E, Tsvetkov V, Fedintsev A, Shaposhnikov M, Krut'ko V, Zhavoronkov A, Kennedy BK. Developing criteria for evaluation of geroprotectors as a key stage toward translation to the clinic. Aging Cell. 2016;15:407-15

49. Shaposhnikov MV, Zemskaya NV, Koval LA, Schegoleva EV, Zhavoronkov A, Moskalev AA. Effects of N-acetyl-L-cysteine on lifespan, locomotor activity and stress-resistance of 3 Drosophila species with different lifespans. Aging (Albany NY). 2018;10:2428-58.

50. Lashmanova E, Zemskaya N, Proshkina E, Kudryavtseva A, Volosnikova M, Marusich E, Leonov S, Zhavoronkov A, Moskalev A. The evaluation of geroprotective effects of selected flavonoids in Drosophila melanogaster and Caenorhabditis elegans. Front Pharmacol. 2017;8:884

51. Danilov A, Shaposhnikov M, Shevchenko O, Zemskaya N, Zhavoronkov A, Moskalev A. Influence of non-steroidal anti-inflammatory drugs on Drosophila melanogaster longevity. Oncotarget. 2015;6:19428-44.

52. Lashmanova E, Proshkina E, Zhikrivetskaya S, Shevchenko O, Marusich E, Leonov S, Melerzanov A, Zhavoronkov A, Moskalev A. Fucoxanthin increases lifespan of Drosophila melanogaster and Caenorhabditis elegans. Pharmacol Res. 2015;100:228-41.

53. Shaposhnikov M, Latkin D, Plyusnina E, Shilova L, Danilov A, Popov S, Zhavoronkov A, Ovodov Y, Moskalev A. The effects of pectins on life span and stress resistance in Drosophila melanogaster. Biogerontology. 2014;15: 113-27. 
54. Barardo D, Thornton D, Thoppil H, Walsh M, Sharifi S, Ferreira S, Anzic A, Fernandes M, Monteiro P, Grum T, Cordeiro R, De-Souza EA, Budovsky A, Araujo N, Gruber J, Petrascheck M, Fraifeld VE, Zhavoronkov A, Moskalev A, de Magalhaes JP. The DrugAge database of aging-related drugs. Aging Cell. 2017;16:594-7.

55. Moskalev A, Chernyagina E, de Magalhaes JP, Barardo D, Thoppil H, Shaposhnikov M, Budovsky A, Fraifeld VE, Garazha A, Tsvetkov V, Bronovitsky E, Bogomolov V, Scerbacov A, Kuryan O, Gurinovich R, Jellen LC, Kennedy B, Mamoshina P, Dobrovolskaya E, Aliper A, Kaminsky D, Zhavoronkov A. Geroprotectors.org: a new, structured and curated database of current therapeutic interventions in aging and age-related disease. Aging (Albany NY). 2015;7:616-28.

56. Zeidler MP, Perrimon N, Strutt DI. Polarity determination in the Drosophila eye: a novel role for unpaired and JAK/STAT signaling. Genes Dev. 1999;13: 1342-53.

57. Ashburner M. Drosophila: a laboratory manual. Cold Spring Harbor, N.Y: Cold Spring Harbor Laboratory; 1989

58. Fleming TR, O'Fallon JR, O'Brien PC, Harrington DP. Modified KolmogorovSmirnov test procedures with application to arbitrarily right-censored data. Biometrics. 1980;36:607-25.

59. Mantel N. Evaluation of survival data and two new rank order statistics arising in its consideration. Cancer Chemother Rep. 1966;50:163-70.

60. Wang C, Li Q, Redden DT, Weindruch R, Allison DB. Statistical methods for testing effects on "maximum lifespan". Mech Ageing Dev. 2004;125:629-32.

61. Han SK, Lee D, Lee H, Kim D, Son HG, Yang JS, Lee SV, Kim S. OASIS 2: online application for survival analysis 2 with features for the analysis of maximal lifespan and healthspan in aging research. Oncotarget. 2016;7: 56147-52.

62. Mallona I, Weiss J, Egea-Cortines M. PcrEfficiency: a Web tool for PCR amplification efficiency prediction. BMC Bioinformatics. 2011;12:404.

63. Livak KJ, Schmittgen TD. Analysis of relative gene expression data using realtime quantitative $P C R$ and the $2^{-\Delta \Delta C(T)}$ method. Methods. 2001;25:402-8.

64. Salomon RN, Jackson FR. Tumors of testis and midgut in aging flies. Fly (Austin). 2008;2:265-8.

Ready to submit your research? Choose BMC and benefit from:

- fast, convenient online submission

- thorough peer review by experienced researchers in your field

- rapid publication on acceptance

- support for research data, including large and complex data types

- gold Open Access which fosters wider collaboration and increased citations

- maximum visibility for your research: over $100 \mathrm{M}$ website views per year

At $\mathrm{BMC}$, research is always in progress.

Learn more biomedcentral.com/submissions 